Original Article

\title{
PHARMACIST INTERVENTION AND PREPARATION OF MANUAL IN THE ADMINISTRATION OF DRUGS THROUGH ENTERAL FEEDING TUBE
}

\author{
L. INDUJA, ASLAM TA, CHITHRA S, G. ANDHUVAN*
}

Affiliated to PSG College of Pharmacy, Department of pharmacy practice, Coimbatore

Email: andhuvangandhi@gmail.com

Received: 01 Jun 2018 Revised and Accepted: 27 Oct 2018

\begin{abstract}
Objective: To monitor errors in current practice and prepare a manual for proper drug administration through the enteral feeding tube.

Methods: It is a prospective observational study conducted for a period of 6 mo in eight departments. The current drug administrations were monitored and checked for the errors which include crushed non crushable solid dosage forms, each drug is not prepared separately, incorrect solution used for dilution, drugs mixed with feeding formula, each drug is not administered separately, not flushed before administration of each drug, not flushed after administration of each drug and others (tablets are not crushed with proper device, motors and pestles are not cleaned frequently, spillage during crushing). Using the data, a manual was prepared and submitted to the physicians of each department.
\end{abstract}

Results: The most prominent error was found to be that the drugs were not prepared and administered separately, tube not flushed before drug administration. Uses of noncrushable tablets were high in neurology. Pantoprazole (enteric coated) and prazosin (modified release) tablets were the most commonly used noncrushable drugs.

Conclusion: The study observed the need for developing a standard protocol for drug administration through enteral feeding tube by the pharmacist along with the physician, nursing team to improve the quality of enteral therapy.

Keywords: Enteral feeding tube, Drug administration, Errors, Standard protocol

(c) 2018 The Authors. Published by Innovare Academic Sciences Pvt Ltd. This is an open access article under the CC BY license (http://creativecommons.org/licenses/by/4.0/) DOI: http://dx.doi.org/10.22159/ijpps.2018v10i12.27655

\section{INTRODUCTION}

Enteral tube feeding (ETF) refers to the delivery of a nutritionally complete feed (containing protein or amino acids, carbohydrate, fat, water, minerals and vitamins) directly into the gut via a tube. It is the preferred method of nutrition support in patients who have a functioning gastrointestinal tract but who are unable to be fed orally. This method of delivering nutrition is also commonly used for administering medications when patients cannot swallow safely [1]. The correct administration of oral drugs to patients on enteral tube feeding presents a special challenge. The key to managing medications in enterally fed patients is to focus on prioritizing therapeutic goals [2].

Giving medications through a feeding tube can be fraught with errors that occur more often than they are recognized and reported. These mistakes are often the result of administering drugs that are incompatible with administration via a tube, of not preparing the medications properly, administering a drug using improper administration techniques. These inaccuracies can result in an occluded feeding tube, a reduced drug effect, or drug toxicity. These potential adverse outcomes can lead to patient harm or even death [3].

The enteric coated medications are designed to prevent drug dissolution in the stomach and to promote absorption in the small intestine. If the tablet is crushed and passed down the enteral feeding tube, undesirable side effects may occur. These could include stomach irritation and a decrease in drug effectiveness. Modified release medications are drugs which are intended to be released gradually over time, and often have a special coating to enable the property. If the tablet is crushed and passed down the enteral feeding tube, an increase in the expected peak plasma level may occur. The patient will be initially exposed to significantly higher-than-normal levels which will increase the chance of side effects [17].

All healthcare professionals especially clinicians and nurses should be aware of the importance of selection of drugs for enteral feed which will help to minimize or prevent the occurrence of errors. The pharmacist play a vital role in minimizing the errors by providing educational programs including preparing evidence-based booklet and classes for case group nurses. An integrated educational program by clinical pharmacists that focus on promoting the correct administration of drugs via enteral feeding catheters significantly improve the knowledge and practice of nurses [4].

Keeping these facts in mind, this study was undertaken to assess the quality of oral drug administration in patients with enteral feeding tubes and to provide a general overview of enteral feeding tube administration, considering dosage form selection. The main aim of the study was to prepare a manual for safe administration of drugs through the enteral feeding tube. Their objective was to monitor drug administration procedure through the enteral feeding tube and to identify errors on the current practice of drug administration through the enteral feeding tube.

\section{MATERIALS AND METHODS}

This is a prospective observational study which was conducted in 8 departments (Cardiology, Gastroenterology, General medicine, General Surgery, Nephrology, Neurology, Neurosurgery, and Paediatrics) of a tertiary care teaching hospital after getting approval from an Institutional Human Ethical Committee with their approval number of $17 / 056$. The patients were selected during the time period of February to July 2017.

Calculation of the sample size was done based upon the number of inpatients with the enteral feeding tube in each department. A total number of 646 drug administrations were monitored in 200 patients based on predetermined inclusion and exclusion criteria. All adult, pediatric and geriatric patients on enteral feeding who receive at least one oral dosage forms through enteral feeding tube were included in the study. Patients receiving nutrition alone through enteral feeding tube were excluded from the study.

A specially designed data collection form was used to enter all study required details like patient name, age, sex, inpatient number, date of admission, date of discharge, department, complaints on admission, type of tube used, drugs prescribed during treatment 
Provision is given in the format to tick the type of error that occurred during drug administration procedure. The current administration procedures were monitored and analyzed to identify errors. The errors include crushed non crushable solid dosage forms, each drug is not prepared separately, incorrect solution used for dilution, drugs mixed with feeding formula, each drug is not administered separately, not flushed before administration of each drug, not flushed after administration of each drug and others (tablets are not crushed with proper device, motors and pestles are not cleaned frequently, spillage during crushing).

The reports were analyzed, documented and then presented. The data entry and statistical analysis were done using software SPSS version 20. Later based of standard guidelines such as American Society for Parenteral and Enteral Nutrition (A. S. P. E. N.), The National Institute for Health and Care Excellence (NICE) etc. and based on observed errors a manual for the proper administration of drugs through enteral feeding tube was prepared and submitted to the physicians and nurses of each department.

\section{RESULTS}

In the study, 646 drug administrations were monitored in 200 patients. Out of total population, $67.5 \%$ constituted male and $32.5 \%$ constituted female. Majority of patients fall in the category 41-60 y (37\%) followed by 61-80 y (34\%), 21-40 y (17\%), 81-100 y (6.5\%) and $0-20 \mathrm{y}(5 \%)$. Majority of drug administrations were observed in the Neurology department followed by General medicine, Gastroenterology and Surgery departments. Among 200 patients, 182 were receiving drugs through the nasogastric tube and 18 patients were receiving drugs through the nasojejunal tube.

A total of 205 oral drugs were observed (184-solid form, 21-liquid form). Solid dosage forms included uncoated (70\%), modified (6\%), enteric coated (2\%) tablets, granules (3\%) and capsules (19\%). Liquid dosage forms included suspensions (33\%), solutions $(10 \%)$, elixirs
(19\%) and syrups (38\%). Out of 128 solid dosage forms, 55 solid dosage forms can be substituted with liquid dosage forms (fig. 1).

The most common error was found to be, not flushed before administration of each drug (99.5\%), each drug not prepared separately (96.5\%), not administered each drug separately (96.5\%). The average number of errors in each prescription was found to be 4.69. Percentage distribution of each error is depicted in table 1 . Noncrushable medications were crushed during several drug administrations and were observed to be a major error (table 2). Most of the modified release and enteric coated tablets crushed during administration are mentioned in fig; 2 and 3 respectively. Among modified release tablets Prazosin was present in a maximum number of prescriptions compared to other modified release tablets and about 79 prescriptions contained pantoprazole enteric-coated tablet. Among capsules, Aspirin+Atorvastatin capsules (EC) was found to be crushed in a maximum number of prescriptions compared to other noncrushable capsules (table 3).

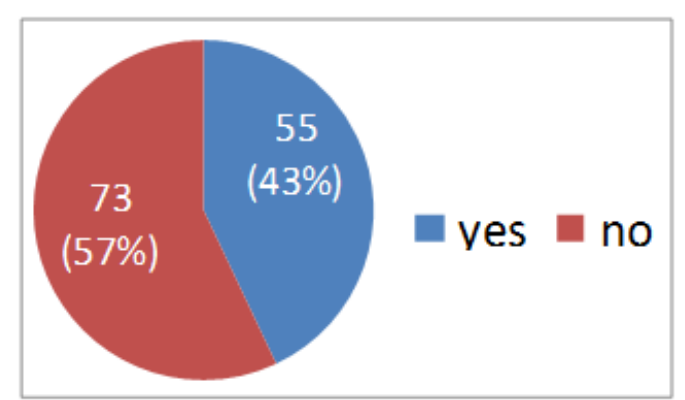

Fig. 1: Availability of alternative liquid dosage forms for solid dosage forms

Table 1: Percentage distribution of errors $(n=646)$

\begin{tabular}{lll}
\hline Type of errors & Number of administrations & Percentage (\%) \\
\hline Each drug is not prepared separately & 623 \\
Crushed noncrushable solid dosage forms & 332 \\
The Incorrect solution used for dilution & 203 \\
Drugs mixed with feeding formula & 138 \\
Not administered each drug separately & 623 \\
Not flushed before administration of each drug & 64.5 \\
Not flushed after administration of each drug & 197 \\
Others & 37.5 \\
\hline
\end{tabular}

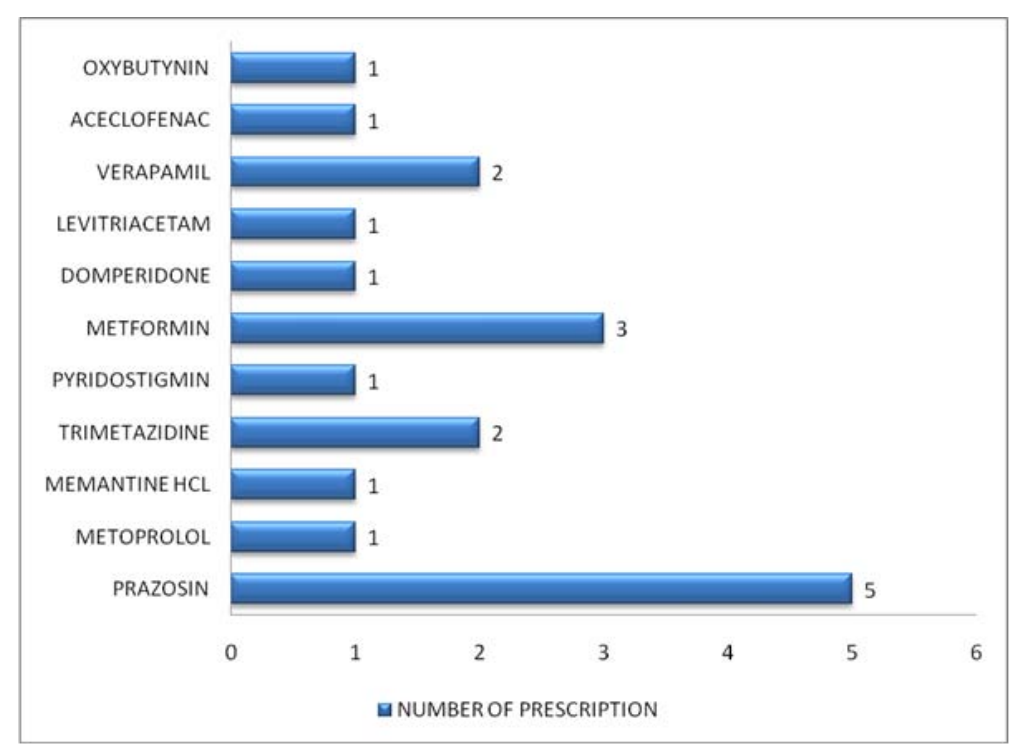

Fig. 2: Number of prescriptions with modified release tablets 
Table 2: Percentage distribution of prescriptions with noncrushable solid dosage forms

\begin{tabular}{|c|c|c|c|c|c|}
\hline \multirow[t]{2}{*}{ Departments } & \multirow[t]{2}{*}{$\mathbf{N}$} & \multicolumn{2}{|c|}{ Number of prescription } & \multicolumn{2}{|c|}{ Percentage (\%) } \\
\hline & & EC & MR & EC & MR \\
\hline Neurology & 96 & 43 & 20 & 44.7 & 20.8 \\
\hline General Medicine & 33 & 11 & 7 & 33.3 & 21.2 \\
\hline Gastroenterology & 24 & 12 & 2 & 50 & 8.3 \\
\hline Neurosurgery & 21 & 9 & 2 & 42.8 & 9.5 \\
\hline Surgery & 19 & 8 & 2 & 42.1 & 10.5 \\
\hline Cardiology & 2 & 1 & 2 & 50 & 100 \\
\hline Pediatrics & 3 & 0 & 0 & 0 & 0 \\
\hline Nephrology & 2 & 2 & 1 & 100 & 50 \\
\hline Total & 200 & 86 & 36 & & \\
\hline
\end{tabular}



Fig. 3: Number of prescriptions with enteric coated tablets

Table 3: Number of prescriptions in individual noncrushable capsules

\begin{tabular}{lll}
\hline Drugs & Number of prescriptions & Percentage (\%) \\
\hline Ambroxol & 2 & 1 \\
Aspirin+atorvastatin & 4 & 2 \\
Clopidogrel+aspirin & 3 & 1.5 \\
Esomeprazole+domperidone & 2 & 1 \\
Indomethacin & 1 & 0.5 \\
Metoprolol & 1 & 0.5 \\
Tamsulosin & 2 & 1 \\
\hline
\end{tabular}

Correlation between the number of drugs prescribed per patient and number of medication errors per patient using Pearson's correlation method was found to statistically significant at p-value of 0.000 .

\section{DISCUSSION}

In our study, a total of 205 oral drugs were used of which 184 were solid dosage forms and 21 were liquid dosage forms. It was found that most of the drugs were administered in solid dosage forms and nearly $43.3 \%$ of them could be substituted by injection or oral liquid formulations, which was supported by the study of Seyed Mojtaba et al., where they concluded that 41 (35.34\%) out of 116 different solid drugs (except effervescent tablets and powders) could be substituted by liquid or injectable forms [18]. A study conducted by Barbosa et al. reported that $72.7 \%$ of studied cases were receiving solid medications, and it was possible that some of the drugs could be substituted by intravenous formulations [12].

Among the administered medicines, pantoprazole was the most frequently prescribed and had the highest percent of wrong dose preparation. Our results were contrary to most studies in Brazil where the liquid dosage form of proton pump inhibitors were used [6-8].

During the prescription analysis, it was observed that some drugs that are critical to the administration via feeding tubes were prescribed, including enteric coated tablets (in 83 patients) controlled release tablets (19 patients) which was found to be consistent with the study conducted by Presoti et al. where 24 patients were prescribed with controlled-release tablets [7].

Among 200 patients, 43(21.5\%) patients received medications mixed with their feed. Our results were inconsistent with the study conducted by Heydrich J et al. were enteral nutrition feed was stopped at the moment of drug administration in majority of the cases [12].

In our study, only $0.5 \%$ of tubes were washed before administration of a drug and $69.5 \%$ of tubes were washed after administration of drugs. A pattern similar to this has been reported in a brazilian study [12]. Out of 50 patients with more prescribed drugs only in 4 patients the drugs were prepared and administered separately in the study of Heydrich J et al. [12] but in our study, nearly $100 \%$ of the patients received drugs which were not prepared and administered separately. It was found that separate preparation and administration of drugs through enteral feeding tube was not observed in $100 \%$ of the cases in this study which was inconsistent with the observations of Heydrich et al. study [12].

The number of drugs prescribed to the patient and the total number of errors occurred to each patient was correlated, which was found to be significant at 0.01 level, from which it can be concluded that as the number of drugs prescribed to each patient increases the risk of errors also increases.

\section{CONCLUSION}

Based on the results obtained during the study and standard guidelines, a manual for the proper administration of drugs through the enteral feeding tube was prepared and submitted the physicians and nurses of each department. The rational approach to decrease 
these inappropriate practices and risks to patients with enteral feeding tubes should include an in-service training program for nursing staffs, and obtaining assistance from the pharmacy service. Close cooperation between medical teams including pharmacists, physicians, and nurses can result in correct administration of drugs through enteral catheters. The study observed the need for developing a protocol for drug administration in patients on enteral therapy along with the physician, nursing team to improve the quality of enteral therapy.

The limitation of their study was the inability to identify the osmolality and sorbitol content of the liquid dosage forms administered through the enteral feeding tube.

S. Chithra, Aslam. T. A, L. Induja were involved in designing the protocol, screening of patients, data collection and monitoring, interpretation of data, maintaining patient file and master file of the project, submission of final report to IHEC and publication. Dr. G. Andhuvan was involved in designing the protocol, interpretation of data, maintaining patient file and publication.

\section{ACKNOWLEDGEMENT}

We extend our gratefulness to Dr. Ritto Thomas, Pharm D, Critical Care Clinical Pharmacist, and all the doctors of Neurology, Neurosurgery, Gastroenterology, Surgery, General Medicine, Paediatrics, Cardiology and Nephrology department for their support and guidance.

\section{AUTHORS CONTRIBUTIONS}

All the author have contributed equally

\section{CONFLICT OF INTERESTS}

Declared none

\section{REFERENCES}

1. Williams NT. Medication administration through enteral feeding tubes. Am J Health Syst Pharm 2008;65:2347-57.

2. Van den Bemt PM, Cusell MB, Overbeeke PW, Trommelen M, Van Dooren D, Ophorst WR, et al. Quality improvement of oral medication administration in patients with enteral feeding tubes. Qual Saf Health Care 2006;15:44-7.

3. Grissinger M. Preventing errors when drugs are given via enteral feeding tubes. P T 2013;38:575-6.

4. Dashti Khavidaki S, Badri S, Eftekharzadeh SZ, Keshtkar A, Khalili H. The role of clinical pharmacist to improve medication administration through enteral feeding tubes by nurses. Int J Clin Pharm 2012;34:757-64.
5. Sohrevardi SM, Jarahzadeh MH, Mirzaei E, Mirjalili M, Tafti AD, Heydari B. Medication errors in patients with enteral feeding tubes in the intensive care unit. J Res Pharm Pract 2017;6:100-5.

6. Barbosa AP, de Paula SL, Barbosa DS, da Cunha DF. Oral drug administration by enteral tube in adults at a tertiary teaching hospital. Eur Soc Parenteral Enteral Nutr J 2012;7:e241-4.

7. Silva MJ, Cava CE, Pedroso PK, Futuro DO. Evaluation of the profile of drug therapy administered through enteral feeding tube in a general hospital in Rio de Janeiro. Braz J Pharm Sci 2011;47:331-7.

8. Presoti AR, do Nascimento MM, Marques LA. Prescription of drugs to be administered through feeding tubes in a Brazilian hospital: profi le and qualifi cation. J Gen Pract 2013;1:112.

9. Heineck I, Bueno D, Heydrich J. Study on the use of drugs in patients with enteral feeding tubes. Pharm World Sci 2009;31:145-8.

10. Emami S, Hamishehkar H, Mahmoodpoor A, Mashayekhi S, Asgharian P. Errors of oral medication administration in a patient with enteral feeding tube. J Res Pharm Pract 2012;1:37-40.

11. NICE Clinical Guidelines, No. 32. Nutrition Support for Adults: Oral Nutrition Support, Enteral Tube Feeding and Parenteral Nutrition. National Collaborating Centre for Acute Care (UK); 2006.

12. Heydrich J, Heineck I, Bueno D. Observation of preparation and administration of drugs by nursing assistants in patients with enteral feeding tube. Braz J Pharm Sci 2009;45:117-20.

13. Bankhead R, Boullata J, Brantley S, Corkins M, Guenter P, Krenitsky J, et al. ASPEN enteral nutrition practice recommendations. J Parenter Enteral Nutr 2009;33:122-67.

14. Beckwith MC, Barton RG, Graves C. A guide to drug therapy in patients with enteral feeding tubes: dosage form selection and administration methods. Hospital Pharm 1997;32:57-64.

15. Belknap DC, Seifert CF, Petermann M. Administration of medications through enteral feeding catheters. Am J Crit Care 1997;6:382-92.

16. White R, Bradnam V. Handbook of drug administration via enteral feeding tubes. $3^{\text {rd }}$ ed. London: Pharmaceutical Press; 2015.

17. Jen Smyth. Senior Pharmacist. editors. The NEWT Guidelines for administration of medication to patients with enteral feeding tubes or swallowing difficulties. 3rd edition. February; 2015. Available from: http://www.newtguidelines.com/ AdminEnteralFeedingTubes.html [Last accessed on 10 Feb 2015].

18. Sohrevardi SM, Jarahzadeh MH, Mirzaei E, Mirjalili M, Tafti AD, Heydari B. Medication errors in patients with enteral feeding tubes in the intensive care unit. J Res Pharm Pract 2017;6:100-5. 